\title{
Effect of Autologous Platelet-rich Plasma on Appearance of Tenocytes at Injured Achilles Tendon Entheses in Rabbits
}

\author{
Tayyaba Faisal1, Rafia Asjid1, Khadija Qamar1, Noreen Akhtar22, Khalida Moeed³ and Tassaduq Hussain 4 \\ 1Department of Anatomy, Army Medical College, Rawalpindi, Pakistan \\ ${ }^{2}$ Armed Forces Institute of Rehabilitation Medicine (AFIRM), Rawalpindi, Pakistan \\ ${ }^{3}$ Department of Anatomy, Loralai Medical College, Loralai, Balochistan, Pakistan \\ ${ }^{4}$ Department of Anatomy, Central Park Medical College, Lahore, Pakistan
}

\begin{abstract}
Objective: To determine the effects of autologous platelet-rich plasma on appearance of tenocytes at injured Achilles tendon entheses in rabbits.

Study Design: Laboratory-based experimental study.

Place and Duration of Study: Department of Anatomy and Pathology, Army Medical College, Rawalpindi, in collaboration with National Institute of Health, Islamabad, from March to May, 2018.

Methodology: Forty healthy male, 4-6 months old New Zealand white rabbits, weighing 2000-2500 gm, were randomly divided into four equal groups A, B, C and D. Group A served as control, while B, C and D were experimental groups. Injury was surgically induced at left Achilles tendon entheses of experimental groups, which were treated with injections of autologous platelet-rich plasma, at the time of induction of injury in group $C$ and two weeks after injury in group $D$, while no treatment was given in group B. Animals were euthanised at the end of 12 weeks. Specimens were taken and processed for histological observations. Bonar's modified score was used to assess the appearance of tenocytes. SPSS version 21 was used for statistical analysis. Chi-square test was applied and $p$-value of $\leq 0.05$ was taken as significant.

Results: Experimental group B showed marked deterioration in Bonar's score as compared to the control group A. The scores were significantly improved in treatment groups $C$ and $D$ as compared to group $B$.

Conclusion: Injection of autologous platelet-rich plasma effectively improved the Bonar's modified score for appearance of tenocytes at injured entheses, 12 weeks after injury.
\end{abstract}

Key Words: Achilles tendon, Tenocytes, Platelet-rich plasma, Growth factors.

How to cite this article: Faisal T, Asjid R, Qamar K, Akhtar N, Moeed K, Hussain T. Effect of autologous platelet-rich plasma on appearance of tenocytes at injured achilles tendon entheses in rabbits. J Coll Physicians Surg Pak 2019; 29(11):1029-33.

\section{INTRODUCTION}

The Achilles tendon or tendo calcaneus is the largest and strongest tendon in the body present on the back of the leg. It attaches the tendons of gastrocnemius and soleus muscles to the calcaneus bone of the heel and causes plantar flexion of foot at the ankle joint. ${ }^{1}$ Enthesis is a transitional zone which attaches tendons and ligaments to bones. Fibrocartilaginous enthesis has four distinct areas; dense regular connective tissue/tendon, uncalcified fibrocartilage, calcified fibrocartilage and the bone. Each zone has its own anatomical, mechanical and physiological characteristics which help in movement at joints and reduce mechanical stress. Collagen fibers are the major constituent of matrix that preserve the structural organization of tissue. Bundles of type 1 collagen fibers, arranged parallel to each other, are

Correspondence to: Dr. Tayyaba Faisal, Department of Anatomy, Army Medical College, Rawalpindi, Pakistan

E-mail: tayyaba_faisal1@yahoo.com

Received: February 06, 2019; Revised: August 07, 2019;

Accepted: August 09, 2019 present in tendon and fibrocartilage areas of enthesis. ${ }^{2}$ The Achilles tendon injuries are among the most frequently occurring disorders of the musculoskeletal system in athletes. ${ }^{3}$ Such injuries at the site of enthesis result in poor healing and long periods of rehabilitation. Various available treatment options range from conservative rehabilitation approach to surgical interventions, but these fail to effectively regenerate it and result in the formation of scar tissue with poor mechanical strength, making it prone to recurrent interventions. ${ }^{4}$

Use of platelet-rich plasma (PRP) is one of the latest treatment modalities, which is under trial in the efforts to promote healing of injured musculoskeletal tissues like tendons, entheses, muscles and bones. PRP has gained popularity because of its easy availability, affordability, simplicity and safety. ${ }^{5}$ PRP contains a multitude of growth factors like platelet-derived growth factor (PDGF), epidermal growth factor (EGF), vascular endothelial growth factor (VEGF), transforming growth factor (TGF), fibroblastic growth factor (FGF), hepatocyte growth factor (HGF) and insulin-like growth factor-1 (IGF-1). ${ }^{6}$ These growth factors interact with the receptors present 
on the surface of cells and cause gene expression which promotes tissue healing by promoting mitotic activity in cells, increased formation of extracellular matrix and decrease in inflammation. 7,8

Many researchers have been investigating the novel uses of PRP in the recent years, but many significant questions regarding effective dose, frequency and appropriate time for administration, different methods, the most suitable area of delivery, and best conditions for use of PRP, demand further scrutiny in this field. 9 PRP's effectiveness at the crucial area of enthesis, has not been a focus in most of the cases. Most of the studies are focused on the regenerative effects of PRP in acutely injured tendon, despite the fact that patients mostly present in chronic phase of injury. 10 This study was conducted to evaluate the regenerative effects of early and delayed injections of autologous PRP, at surgically injured Achilles tendon enthesis (ATE) in rabbits, 12 weeks after injury, using appearance of tenocytes as the determining marker.

\section{METHODOLOGY}

This was a laboratory-based experimental study, carried out in the Department of Anatomy and Pathology, Army Medical College, Rawalpindi, in association with National Institute of Health (NIH), Islamabad. The approval for the study was taken from Ethical Review Committee of the Army Medical College, Rawalpindi and National University of Medical Sciences, Islamabad.

Non-probability convenience sampling technique was used. Forty healthy male, 4-6 months old New Zealand white rabbits having $2000-2500$ gm body weight, were selected. They were kept in separate cages at controlled room temperature of $20-25^{\circ} \mathrm{C}$ and 12 -hour light and dark cycles were maintained throughout the experimental period. Standard laboratory diet and water was given ad libitum.

Animals were randomly distributed into four equal groups $A, B, C$ and $D$, having 10 rabbits in each group. Group A served as control. Injury was surgically induced in experimental groups B, C and D. Anesthesia was given to animals by using intramuscular injection of a mixture of xylazine and ketamine hydrochloride (xylazine: $5 \mathrm{mg} / \mathrm{kg}$ and ketamine: $35 \mathrm{mg} / \mathrm{kg}$ body weight). ${ }^{11} \mathrm{~A} 2 \mathrm{~cm}$ long skin incision was given on the left hind legs of rabbits at the region of ATE. Injury was produced by a punch biopsy instrument (Sklar, $2 \mathrm{~mm}$ Disposable Biopsy Punch) in the middle of the ATE. Skin was closed with a disposable skin stapler (Advan). No treatment was given to animals in group B. Injection of PRP was administered in ATE of rabbits of group $C$ at the time of induction of injury. Animals in group $D$ received injection two weeks after injury. Free mobility was allowed to all the animals and examinations were done twice daily to look for any untoward effects of surgical procedure.
Platelet-rich plasma was prepared by drawing $10 \mathrm{ml}$ of whole blood from the marginal ear vein of each rabbit using a 21-gauge needle. Blood was mixed with $1 \mathrm{ml}$ of $0.1 \mathrm{M}$ sodium citrate in a Falcon $15 \mathrm{ml}$, conical centrifuge tube. To perform baseline platelet count, $1 \mathrm{ml}$ of whole blood was kept in a separate tube. 12

A standard laboratory centrifuge (Hettich EBA 20) was used for centrifugation. First spin was done at $500 \mathrm{~g}$ for 10 minutes. ${ }^{13}$ It yielded three distinct layers. Plateletpoor plasma (PPP) was present in the top most layer, platelets and white blood cells were there in the middle buffy coat, and red blood cells were found in the bottom layer. The top most and middle layers were gently aspirated and shifted to a new Falcon tube.

A second spin was given at $2200 \mathrm{~g}$ for a period of 10 minutes for further concentration of PRP. ${ }^{13}$ Supernatant or PPP was discarded after removing it by gentle aspiration. Platelets were separated in the pellet present at the bottom of the tube. Gentle shaking was done to resuspend the pellet. Then it was recovered along with a small volume of PPP and was called as PRP. ${ }^{13}$ Platelets count was performed both in the whole blood and PRP by automated cell counter. Platelets concentration of about three times in PRP above the baseline value of whole blood was utilised for injections to stimulate regeneration in injured ATE. PRP was activated by using $500 \mu \mathrm{l}$ of $10 \% \mathrm{CaCl}_{2}$ solution. 14 Injections of PRP were administered within an hour of its preparation to utilize maximum concentration of growth factors. ${ }^{8}$

Total duration of the experimental period was 12 weeks. At the end of this period, all animals were euthanised by intravenous injection of pentobarbital $(30 \mathrm{mg} / \mathrm{kg}$ ) followed by an intramuscular injection of $2 \%$ xylazine $(5 \mathrm{mg} / \mathrm{kg}) .{ }^{15}$ Animals were dissected and specimens of ATE were taken. After decalcification in $5 \%$ nitric acid, samples were processed and embedded in paraffin. Rotary microtome was used to cut $5 \mu \mathrm{m}$ thick longitudinal sections. Sections were stained with hematoxylin and eosin stains. Each slide was examined under light microscope at 10X and 40X magnifications. Area of the most prominent change was chosen for recording the observations. ${ }^{16}$

Bonar's modified semi-score for tendon lesion determination was used to evaluate the appearance of tenocytes. ${ }^{15}$ According to Bonar's score, grading was done from 0-3: grade 0 was completely normal cell with elongated spindle shaped nucleus and inconspicuous cytoplasm; grade 1 was an increased roundness of the nucleus with clear cytoplasm; grade 2 was an increased roundness and size of cell with round nucleus, and a small amount of cytoplasm was visible; grade 3 was a large cell with a round nucleus, abundant cytoplasm, and lacuna formation (chondroid change). ${ }^{15}$

Data analysis was done using the statistical package for social sciences (IBM-SPSS version 21). Chi-square test 
Table I: Frequencies and percentages of different appearances of tenocytes of control group A and experimental groups B, C and D.

\begin{tabular}{l|c|c|c|c|c}
\hline Parameter & Findings & Group A & Group B & Group C & Group D \\
\hline Appearance of tenocytes & Grade 0 & $10(100 \%)$ & $0(0.0 \%)$ & $7(70 \%)$ & $5(50 \%)$ \\
& Grade 1 & $0(0.0 \%)$ & $1(10 \%)$ & $3(40 \%)$ & $5(50 \%)$ \\
& Grade 2 & $0(0.0 \%)$ & $6(60 \%)$ & $0(0.0 \%)$ & $0(0.0 \%)$ \\
& Grade 3 & $0(0.0 \%)$ & $3(30 \%)$ & $0(0.0 \%)$ & $0(0.0 \%)$ \\
\hline
\end{tabular}

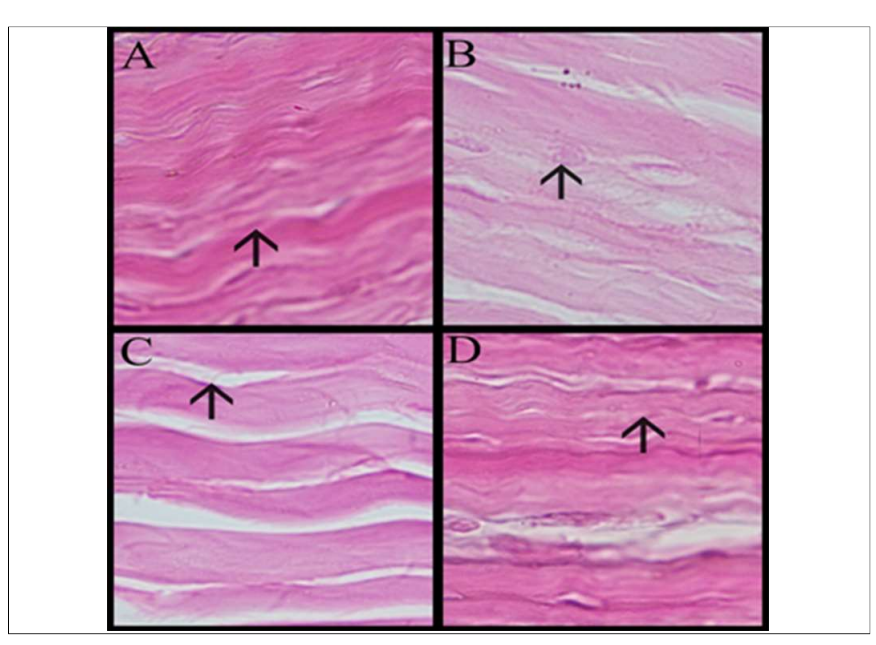

Figure 1: Photomicrographs showing appearance of tenocytes in control group $A$ and experimental groups $B, C$ and $D$, arrows indicating tenocytes, at 40X; H\&E.

was applied and results were expressed as frequencies and percentages. A $p$-value of $\leq 0.05$ was considered significant.

\section{RESULTS}

All rabbits remained alive, healthy and active till the end of the experimental period. Microscopic examination of all the sections was done under light microscope at $10 \mathrm{X}$ and 40X magnification. Appearance of tenocytes was studied by using modified Bonar's score. Area of the most prominent change was selected for evaluation. Scoring was done from $0-3$, with ' 0 ' being completely normal and ' 3 ' being maximally abnormal. In control group A, tenocytes showed totally normal appearance having inconspicuous elongated spindle shaped nuclei with no obvious cytoplasm, in all of the animals (Table I, Figure 1).

In experimental group B, $6(60 \%)$ animals had moderate abnormality (grade $=2$ ) with increased roundness and size; the nuclei were round and slightly enlarged and a small amount of cytoplasm was visible, 3 animals (30\%) had severe abnormality (grade $=3$ ) with round nuclei, large cells with abundant cytoplasm and lacuna formation (chondroid change) and one rabbit (10\%) showed mild (grade $=1$ ) changes with increased roundness of the nuclei and clear cytoplasm (Table I, Figure 1). In experimental group $C, 7(70 \%)$ animals showed normal appearance of tenocytes and $3(30 \%)$ had grade 1 abnormality (Table I, Figure 1). Experimental group D, had $5(50 \%)$ animals with normal appearance of tenocytes and $5(50 \%)$ had mild abnormality (Table I, Figure1).
On intergroup comparison, it was seen that difference between experimental group $B$ and control group $A$ was highly significant $\left(p=0.001^{*}\right)$. Experimental Group $D$ was also significantly different from group $A(p=0.010)$, but the difference between group $C$ and $A$ was not statistically significant $(p=0.060)$. When group $B$ was compared to groups $C$ and $D$, the $p$-value was 0.001 in each case. On intergroup comparison of groups $C$ and $D$, $p$-value was statistically insignificant $(p=0.361)$.

\section{DISCUSSION}

Tendon pathologies are among the common health issues involving about $8 \%$ of the population. They end up in limited and painful mobility leading to physical, mental and financial stress. ${ }^{17}$ Achilles tendon is the most frequently injured tendon in human body. There is an increased risk of reinjures after such pathologies. ${ }^{18}$ ATE is difficult to regenerate because of the fibrocartilage. 13 Different treatment modalities are being used to restore injured tendon, such as surgical procedures, chemical methods and tissue engineering techniques but, pursuit for the ideal mode of management is still continuous. ${ }^{19}$

One of the latest methods to regenerate tendons and damaged enthesis, is the use of PRP. ${ }^{5}$ PRP is a natural product of the blood plasma, having platelets in a concentration more than the baseline value of the whole blood.20 Numerous growth factors are present in platelets that play a crucial part in regeneration of tissues. These exert therapeutic effect by stimulating proliferation of cells like fibroblasts, tenocytes, chondrocytes and mesenchymal stem cells (MSCs), which in turn increase production of matrix. ${ }^{21}$

The purpose of this research was to observe the effects of early and delayed injections of PRP on appearance of tenocytes at injured ATE in rabbits. Migration and subsequent maturation of tenocytes is one of the imperative parameters to assess healing of tendon injury. Bonar's modified score was used to determine the healing of the dense regular connective tissue/tendon zone of ATE. Number of tenocytes is greatly diminished after injury due to the decreased expression of scleraxis (Scx) gene which leads to programmed death of these cells. ${ }^{22}$ During the stage of inflammation, cells of acute inflammation invade the site of injury. Necrotic material is phagocytosed by macrophages. During the stage of proliferation, tenocytes begin to migrate not only from the surrounding paratendineum and synovial sheath but also from epitendineum and endotendineum. ${ }^{10}$ 
Numerous large tenocytes having rounded nuclei and abundant cytoplasm are seen in initial stages of healing. They produce extracellular matrix and are progressively substituted by cells possessing inconspicuous spindle shaped nuclei and no apparent cytoplasm.

In experimental group B of present study (which did not receive PRP injection): $60 \%$ of the animals had moderate abnormality; $30 \%$ had maximal abnormality and; $10 \%$ showed mild abnormality in appearance of tenocytes, thus proving that all these animals did not have optimal tendon healing. Experimental group C received early PRP injections and $70 \%$ animals had completely normal looking tenocytes while $30 \%$ showed mild abnormality in appearance, thus supporting the ameliorative effect of PRP on injured tendon. Experimental group D received delayed injection of PRP and $50 \%$ of the rabbits depicted normal appearance of tendon cells while other $50 \%$ had mild abnormality in tenocytes. These results also support the healing effect of PRP in this group as compared to the non-treatment group.

This study was in accordance with the research carried out by Gonzalez and colleagues who studied the healing effect of PRP on collagenase-induced degeneration of Achilles tendon in a rabbit model. They found that PRP was beneficial in promoting regeneration of tendons as compared to placebo, by up-regulation of the expression of collagen and down-regulation of expression of cytokines having catabolic effects, thus improving scores for tendon healing. They also suggested the use of multiple injections of PRP for maximum benefits. ${ }^{15}$ Findings of the present study were also in line with observations of Fukawa and other researchers who used Achilles tendon transection model in New Zealand White rabbits to evaluate the effects of PRP. They also used Bonar's grade to study regeneration in tendons. They observed that appearance of tenocytes gradually improved with time in tendons treated with PRP as compared to non-treated animals. They proved the ameliorative effects of PRP on tendon healing. ${ }^{16}$

A research was carried out to see the histological changes in proximal and distal stump of Achilles tendon after its transection. It was demonstrated in this study that epitenon cells from the surrounding healthy tendon shifted towards the site of injury; initially they were large and deep staining cells which gradually infiltrated in between the bundles of collagen fibers and their size and staining was gradually reduced with time as they matured.23 This study also confirmed the findings of Bagheri and colleagues who used PRP alone and in combination with MSCs derived from bone marrow to compare their effects on healing of Achilles tendon injury in rabbits. They found that PRP alone, or in combination with MSCs, improved and shortened the healing process, and scores for tendon healing, including the appearance of tenocytes in treatment groups. ${ }^{24}$ Efficacy of intratendinous injection of PRP to improve the Bonar's modified score for tenocytes in injured Achilles tendon was also confirmed by a study conducted in 2013.25

Thus, it can be established from this study that a number of growth factors present in autologous PRP helped in improving the appearance of tenocytes in experimental models of injured ATE, who were treated with PRP injections as compared to non-treatment group.

\section{CONCLUSION}

The present study concludes that injection of autologous PRP, by virtue of its multitude of growth factors, affects the histomorphology of injured ATE in experimental models, 12 weeks after injury. It causes significant improvement in Bonar's modified score for appearance of tenocytes. PRP injections were found to be equally useful in early and delayed treatment groups as compared to non-treatment group.

\section{DISCLOSURE:}

This article is based on one of the authors' M Phil. Thesis.

\section{FUNDING:}

Authors are highly indebted to National University of Medical Sciences, Islamabad for providing funds for this project.

\section{ETHICAL APPROVAL:}

The approval for the study was taken from Ethical Review Committee of the Army Medical College, Rawalpindi and, National University of Medical Sciences, Islamabad.

\section{CONFLICT OF INTEREST:}

Authors declared no conflict of interest.

\section{AUTHORS' CONTRIBUTION:}

TF: Conception and design of the work; the acquisition, analysis and interpretation of data for the work; drafting the work; critical revision of the work; agreement to be accountable for all aspects of the work in ensuring that questions related to the accuracy or integrity of any part of the work are appropriately investigated and resolved.

RA, NA, KM: Design of the work; contribution in the acquisition and analysis of data for the work; agreement to be accountable for all aspects of the work in ensuring that questions related to the accuracy or integrity of any part of the work are appropriately investigated and resolved. $\mathrm{KQ}$ : Contribution to the conception and design of the work; critical revision of the work; final approval of the version to be published; agreement to be accountable for all aspects of the work in ensuring that questions related to the accuracy or integrity of any part of the work are appropriately investigated and resolved. 
TH: Conception of the work; critical revision of the work; agreement to be accountable for all aspects of the work in ensuring that questions related to the accuracy or integrity of any part of the work are appropriately investigated and resolved.

\section{REFERENCES}

1. Han M, Larson PEZ, Liu J, Krug R. Depiction of achilles tendon microstructure in vivo using high-resolution 3-dimensional ultrashort echo-time magnetic resonance imaging at $7 \mathrm{~T}$. Invest Radiol 2014; 49:339-45.

2. Apostolakos J, Durant TJ, Dwyer CR, Russell RP, Weinreb JH, Alaee $\mathrm{F}$, et al. The enthesis: A review of the tendon-to-bone insertion. Muscles Ligaments Tendons J 2014; 4:333-42.

3. Oryan A, Alidadi S, Moshiri A. Application of collagen implants in achilles tendon injuries. J Sports Med Doping Stud 2015; 6:2161-73.

4. Thevendran G, Sarraf K, Patel N, Sadri A, Rosenfeld P. The ruptured Achilles tendon: A current overview from biology of rupture to treatment. Musculoskelet Surg 2013; 97:9-20.

5. Circi E, Akman YE, Sukur E, Bozkurt ER, Tuzuner T, Ozturkmen Y. Impact of platelet-rich plasma injection timing on healing of Achilles tendon injury in a rat model. Acta Orthop Traumatol Turc. 2016; 50:366-72.

6. Zhang J, Wang JHC. PRP treatment effects on degenerative tendinopathy: An in vitro model study. Muscles Ligaments Tendons J 2014; 4:10-7.

7. Zhang J, Middleton KK, Fu FH, Im H-J, Wang JH. HGF mediates the anti-inflammatory effects of PRP on injured tendons. PLoS One 2013; 8:e67303.

8. Guszczyn T, Surazynski A, Zareba I, Rysiak E, Popko J, Palka J. Differential effect of platelet-rich plasma fractions on $\beta 1$-integrin signaling, collagen biosynthesis, and prolidase activity in human skin fibroblasts. Drug Des Devel Ther 2017; 11:1849-57.

9. Jeong D, Lee CR, Lee J, Pak J, Kang LW, Jeong B, et al. Clinical applications of platelet-rich plasma in patellar tendinopathy. Biomed Res Int 2014; 2014:249498.

10. Yang G, Rothrauff BB, Tuan RS. Tendon and ligament regeneration and repair: Clinical relevance and developmental paradigm. Birth Defects Res C Embryo Today 2013; 99:203-22.

11. Mahdi AK, Eesa MJ, Khalaf $\mathrm{OH}$. Enhancing Achilles tendon healing by using autologous bone marrow in rabbits. ALQadisiyah J Vet Med Sci 2014; 13:63-70.

12. Nagata MJH, Messora MR, Furlaneto FAC, Fucini SE, Bosco AF, Garcia VG, et al. Effectiveness of two methods for preparation of autologous platelet-rich plasma: An experimental study in rabbits. Eur J Dent 2010; 4:395-402.

13. Zhang J, Yuan T, Zheng N, Zhou Y, Hogan MV, Wang JH. The combined use of kartogenin and platelet-rich plasma promotes fibrocartilage formation in the wounded rat Achilles tendon entheses. Bone Joint Res 2017; 6:231-44.

14. Cavallo C, Roffi A, Grigolo B, Mariani E, Pratelli L, Merli G, et al. Platelet-rich plasma: The choice of activation method affects the release of bioactive molecules. Biomed Res Int 2016; 2016:6591717.

15. González JC, López C, Álvarez ME, Pérez JE, Carmona JU. Autologous leukocyte-reduced platelet-rich plasma therapy for Achilles tendinopathy induced by collagenase in a rabbit model. Sci Rep 2016; 6:19623.

16. Fukawa T, Yamaguchi S, Watanabe A, Sasho T, Akagi R, Muramatsu $Y$, et al. Quantitative assessment of tendon healing by using MR T2 mapping in a rabbit Achilles tendon transection model treated with platelet-rich plasma. Radiology 2015; 276: 748-55.

17. Jafari L, Vachon P, Beaudry F, Langelier E. Histopathological, biomechanical, and behavioral pain findings of Achilles tendinopathy using an animal model of overuse injury. Physiol Rep 2015; 3.e12265.

18. Walden G, Liao X, Donell S, Raxworthy MJ, Riley GP, Saeed A. A clinical, biological, and biomaterials perspective into tendon injuries and regeneration. Tissue Eng Part B Rev 2017; 23:44-58.

19. Sen B, Guler S, Cecen B, Kumtepe E, Bagriyanik A, Ozkal S, et al. The effect of autologous platelet-rich plasma in the treatment of Achilles tendon ruptures: An experimental study on rabbits. Balkan Med J 2016; 33:94-101.

20. Alves R, Grimalt R. A review of platelet-rich plasma: History, biology, mechanism of action, and classification. Skin Appendage Disord 2018; 4:18-24.

21. Xie X, Zhang C, Tuan RS. Biology of platelet-rich plasma and its clinical application in cartilage repair. Arthritis Res Ther 2014; 16:204.

22. Freedman BR, Gordon JA, Soslowsky LJ. The Achilles tendon: Fundamental properties and mechanisms governing healing. Muscles Ligaments Tendons J 2014; 4:245-55.

23. Al-Qattan MM, Mawlana OH, Ahmed RA-TM, Hawary K. Histological changes in the proximal and distal tendon stumps following transection of achilles tendon in the rabbits. $J$ Coll Physicians Surg Pak 2016; 26:349-52.

24. Bagheri F, Tavasoly A, Dehgahan M, Sasani F, Raji M, Mohajeri S. Comparison of platelet-rich plasma (PRP), bone marrowderived mesenchymal stem cells and their combination on the healing of Achilles tendon in rabbits. Iranian J Vet Surg 2017; 12:44-52.

25. Dallaudière B, Lempicki M, Pesquer L, Louedec L, Preux PM, Meyer $\mathrm{P}$, et al. Efficacy of intra-tendinous injection of plateletrich plasma in treating tendinosis: Comprehensive assessment of a rat model. Eur Radiol 2013; 23:2830-7. 